\title{
High-Energy Gamma Rays from the Milky Way: Time-Dependent Modelling of Cosmic Ray Propagation and Non-Thermal Emissions with the GALPROP Code
}

\section{Troy A. Porter*}

W. W. Hansen Experimental Physics Laboratory and Kavli Institute for Particle Astrophysics and Cosmology, Stanford University, Stanford, CA 94305, USA E-mail:

tporterastanford.edu

\section{Guơlaugur Jóhannesson}

Science Institute, University of Iceland, IS-107 Reykjavik, Iceland and AlbaNova Univ. Center Nordita, Roslagstullsbacken 23, SE-106 91 Stockholm, Sweden

E-mail: gudlaugu@hi.is

\section{Igor V. Moskalenko}

W. W. Hansen Experimental Physics Laboratory and Kavli Institute for Particle Astrophysics and Cosmology, Stanford University, Stanford, CA 94305, USA

E-mail: imosestanford.edu

\begin{abstract}
Standard modelling of the distribution of cosmic rays (CRs) throughout the Galaxy is based on a steady-state assumption, where the source spatial density is described using a smoothly varying function of position that does not evolve with time. While this is a convenient approximation, reality is otherwise where primary CRs are produced in and about highly localised regions, e.g., supernova remnants, which have a finite lifetime. With the latest version of the GALPROP code it is now possible to model the injection of CRs over a long time history from a distribution of discrete sources (in space and time) at high spatial resolution and calculate the associated nonthermal emissions from radio to high-energy gamma rays. This paper describes such modelling using time-dependent 3D CR source distributions and 3D models for the interstellar gas, radiation, and magnetic fields with the GALPROP code. The results presented in this paper have relevance for connecting data collected by $\mathrm{GeV}$ energy range instruments, such as the Fermi Large Area Telescope, with observations made at TeV energies by Cherenkov Telescopes, such as HESS, and the forthcoming CTA.
\end{abstract}

36th International Cosmic Ray Conference -ICRC2019-

July 24th - August 1st, 2019

Madison, WI, U.S.A.

${ }^{*}$ Speaker. 


\section{Introduction}

The sky brightness is dominated over a broad range of wavelengths by the non-thermal emissions of cosmic ray (CR) particles that interact with the gas, radiation, and magnetic fields in the interstellar medium (ISM) of the Galaxy. These interstellar emissions present a strong foreground for detection of non-thermal point sources, diffuse signals of exotic origin (e.g., the dark matter), as well as all manner of extragalactic phenomena. They present also a valuable tool for understanding CR sources, the CR injection and propagation through the ISM, and the other constituents of the ISM (gas, radiation, and magnetic fields).

High-quality data tracing the non-thermal emissions now exist, or will be shortly available, over a broad range of the electromagnetic spectrum. The WMAP and Planck instruments have provided comprehensive all-sky data at microwave frequencies, while the forthcoming eROSITA mission [1] will make a sensitive all-sky survey at X-ray energies. These instruments allow tracing the CR electrons/positrons over a broad range of energies and spatial scales via their interactions with magnetic fields in the Galaxy. The Fermi Large Area Telescope [2], launched in 2008, has provided the most comprehensive view of the Galactic high-energy $\gamma$-ray emissions from $\sim 30 \mathrm{MeV}$ up to hundreds of $\mathrm{GeV}$ energies and higher. This energy coverage spans the range from where the smoothly varying CR "sea" is dominating to energies tracing where the localised individual CR source regions are thought to be the most intense contributions, and traces the interactions of both nuclei and electrons with the gas and radiation fields in the Galaxy. The recent release of the HESS Galactic plane survey [3] extends to energies $\gtrsim 1 \mathrm{TeV}$ and shows localised, extended regions embedded in lower intensity broadly distributed emissions - similar, but not the same to the Fermi-LAT data at lower energies, which is much more smoothly distributed. Meanwhile, the HAWC experiment extends coverage of portions of the Galactic plane up to $\sim 100 \mathrm{TeV}$ energies showing that the emissions are dominated by highly localised regions [4]. The complementarity of these multi-wavelength data for probing the CRs and sources across the Galaxy has been anticipated by many authors (e.g., [5, 6]).

Modelling the non-thermal interstellar emissions relies on determination of the CR distribution throughout the Galaxy. The standard approach employs a steady-state assumption for the CR source spatial density described as a smoothly varying function of position that does not evolve with time. This readily facilitates $\mathrm{CR}$ propagation calculations using analytical and numerical methods. For the $\sim 0.1-1 \mathrm{GeV} /$ nuc energy data that are used to constrain model parameters (diffusion coefficient, halo size, etc.), the long CR residence times are thought to provide sufficient mixing to effectively erase individual contributions of the CR sources. This CR "sea" is correspondingly smoothly spatially distributed, thus motivating the initial assumption for the source density.

Reality is, however, otherwise with primary CRs produced in and about highly localised regions, e.g., supernova remnants (SNRs), which have finite lifetimes. The injection of CRs into the ISM by an ensemble of discrete (spatial/temporal) sources has been investigated since the late 1960s (e.g., [7, 8, 9, 10, 11, 12, 13]). However, the connection between such stochastic CR source models and the associated non-thermal emissions have been relatively unexplored. An early version of the GALPROP code was used for preliminary calculations [14, 15], but the data quality at the time and computational limitations meant that the modelling provided limited insight.

This paper describes predictions for the CR intensities and corresponding non-thermal emis- 
sions from the Galaxy made using a time/space-discretised CR source model, and makes a comparison with the equivalent steady-state/continuous solution. Full 3D models for the CR sources and ISM components (gas, radiation field, magnetic field) are used. The comparison is relevant because localised source signatures may be evident in the high-quality data now available, but all analysis to date of high-energy $\gamma$-ray data employs the steady-state models.

\section{Modelling Setup}

The CR propagation and interstellar emission calculations are made using the latest release of the GALPROP code (http://galprop.stanford.edu; [16, 17]). Theoretical understanding of CR propagation in the ISM is the framework that the GALPROP code is built around. The key idea is that all CR-related data, including direct measurements, $\gamma$-rays, sychrotron radiation, etc., are subject to the same physics and must therefore be modelled simultaneously. The GALPROP code numerically solves the system of partial differential equations describing the particle transport with a given source distribution and boundary conditions for all species of CRs. Propagation is described using the diffusion-reacceleration equation, where the processes involved include diffusive reacceleration and, for nuclei, nuclear spallation, secondary particle production, radioactive decay, electron capture and stripping, electron knock-on, and electron K-capture, in addition to energy loss from ionisation and Coulomb interactions. For CR electrons and positrons, important processes are the energy losses due to ionisation, Coulomb scattering, bremsstrahlung (with the neutral and ionised gas), Compton scattering, and synchrotron emission.

Enhancements to the GALPROP code have been implemented to enable more efficient timedependent CR propagation and interstellar emissions modelling. The "discrete sampler" is a new facility that produces a spatial and temporal discretised list of CR source regions from a usersupplied smooth CR spatial density distribution and time interval. This enables direct comparison of GALPROP predictions for time/space discretised realisation and the steady-state solution from the same CR smooth density distribution. The discrete sampler uses an acceptance/rejection method with pseudo random number generator, which allows full reproducibility of the discretisation of the smooth density model for different CR source injected luminosity evolution scenarios for the discrete case. The GALPROP code also includes an option to use non-equidistant grids that allow for increased spatial resolution over user-specified regions. This addition to GALPROP is inspired by the the Pencil Code [18] (see Section 5.4 of http://pencil-code.nordita.org/doc/manual.pdf), where the usage of analytic functions can have advantages in terms of speed and memory usage compared to purely numerical implementations for non-uniform grid spacing.

Without loss of generality, this paper uses the SA50 model described by [16] and [17] for the smooth CR density distribution: it assumes that $50 \%$ of the injected CR luminosity is provided by a 'disc' and the other 50\% is from a 4-arm spiral. The CR source scale height is taken to be $200 \mathrm{pc}$. The R12 interstellar radiation field model developed by [16] is used for the CR electron energy losses and $\gamma$-ray production via IC scattering. Meanwhile, CR electron synchrotron losses/radiation production use the bisymmetric spiral model of [19] for the regular component of the magnetic field together with a $4 \mu \mathrm{G}$ random component.

The propagation model parameters are as determined by [20]. The tuning procedure follows that of $[16,17]$ where the halo size is set to $6 \mathrm{kpc}$ and the parameters are adjusted to reproduce the 
CR data. Solar modulation is accounted for by using the force-field approximation, one modulation potential value for each observation period. The propagation parameters are first determined by fitting $\mathrm{CR}$ spectral data for $\mathrm{Be}, \mathrm{B}, \mathrm{C}, \mathrm{O}, \mathrm{Mg}, \mathrm{Ne}$, and $\mathrm{Si}$. These are then kept fixed and the injection spectra for electrons, protons, and He fitted separately. The procedure is then iterated until convergence.

The model calculations use a 3D right-handed spatial grid with the solar system on the positive $X$-axis and $Z=0 \mathrm{kpc}$ defining the Galactic plane employing the IAU recommended $R_{S}=8.5 \mathrm{kpc}$ for the distance from the Sun to the Galactic centre (GC). A non-uniform spatial grid is used following [20] where the parameters of the transforms for the $X$ and $Y$ coordinates are chosen so that the resolution nearby the solar system is $\sim 50 \mathrm{pc}$, increasing to $\sim 0.5 \mathrm{kpc}$ at the boundary of the Galactic disc, which is at $20 \mathrm{kpc}$ from the GC. In the Z-direction the resolution is $25 \mathrm{pc}$ in the plane, increasing to $0.5 \mathrm{kpc}$ at the boundary of the grid at $\left|Z_{\text {halo }}\right|=6 \mathrm{kpc}$. The energy grid is logarithmic from $100 \mathrm{MeV}$ to $10 \mathrm{TeV}$ with 32 energy planes. This range covers the CR energies that are responsible for producing the non-thermal emissions detected by the Fermi-LAT and current Cherenkov instruments, as well as radio-microwave synchrotron emissions. The upper limit is set also due to physical considerations related to the size of the discrete CR source regions (see below).

For the discrete source CR propagation calculations the model parameters determined using the tuned steady-state solution are employed. The simulation epoch is set to $600 \mathrm{Myr}$, which is a factor $\sim 10$ longer than the dominant (propagation) time scale around a few $\mathrm{GeV}$. The size of an individual source volume is set to be $50 \mathrm{pc}$ in $X, Y$, and $Z$ coordinates, with frequency $1 / 100 \mathrm{yr}$ and active time $10^{5} \mathrm{yr}$ injecting $\mathrm{CR}$ particles at constant luminosity with the same spectral parameters as the smooth density distribution. A fixed timestep of $5 \mathrm{kyr}$ is employed for the solution, which is small enough to capture the propagation and energy losses at the upper boundary of the energy grid described above. The size of the source volumes is chosen to correspond with the approximate physical dimensions where the CR propagation becomes 'ISM-like'; below this the propagation is likely characterised by local effects about the true CR sources rather than in the general ISM [21, 22, 23]. The frequency and active time are chosen as representative of 'SNR'-like sources in the Galaxy without making an identification with a specific class of objects. Only protons and electrons are injected for the discrete source modelling, because they are the primary CR species of major interest for producing also secondary electromagnetic emissions. Using the same spectral parameters and constant injection rate over the active time for a discrete source region provides an equivalent comparison with the results of the steady-state modelling.

\section{Results}

Figure 1 shows the fractional residuals with the steady-state energy density for CR protons (left) and electrons (right) in the Galactic plane and for the $X / Z$ plane for $Y=0 \mathrm{kpc}$ at $1.6 \mathrm{TeV}$. For any sampling interval the 'snapshot' of the discrete source energy density distribution will reflect the recently active and ongoing injection of CRs, together with the remnant contributions by formerly active sources. The non-smoothness caused by this history is clearly seen in the fractional residuals for both species, but the fluctuations are more prominent for the electrons reflecting the much faster energy losses they experience at high energies. Although not shown, fluctuations in the energy density distributions occur also even down to $\sim$ few $\mathrm{GeV}$ energies, although they are not 
as pronounced as those shown for the $1.6 \mathrm{TeV}$ energy particles in Fig. 1. For the electrons the low energy fluctuations are still numerous and widely distributed indicating that a smooth CR 'sea' for these particles, as might be defined by the steady-state solution at $\sim \mathrm{GeV}$ energies, is a debatable notion when the CR sources are discretised spatially and temporally. Looking at the $X / Z$ slice reveals asymmetrical residuals above/below the Galactic plane, which is a result of the sampling from the continuous CR source density distribution. Hence, even though the modelling uses a $Z=0 \mathrm{kpc}$ symmetric source density distribution the finite statistics means that at any moment over the simulation epoch there is no guarantee that the discrete source regions will also be distributed symmetrically about the Galactic plane.
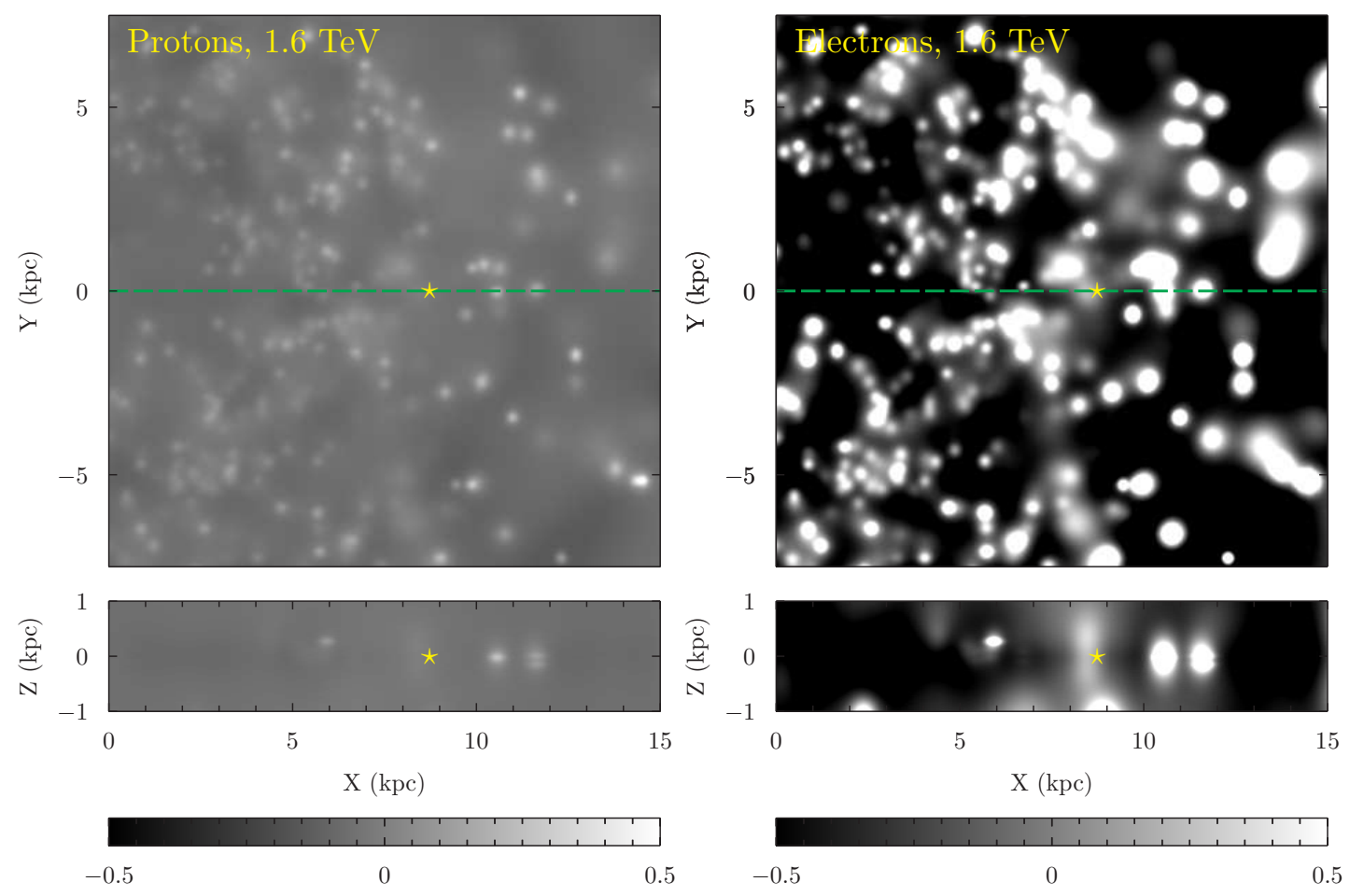

Figure 1: CR proton (left) and electron (right) energy density fractional residuals for the discrete source solution at the end of the simulation epoch with respect to the steady-state case at $1.6 \mathrm{TeV}$. Solar system location is marked by yellow star. The dashed line for each $X / Y$ panel shows location of corresponding slice in $X / Z$ plane shown below it for each particle species and energy.

The CR density variations for the discretised model are across a range of spatial scales that are energy dependent because, even though individual source region properties are uniform, at any time their distribution is due to the cumulative CR injection and activity history modified by propagation and energy losses. The discretised model CR density variations will result also in fluctuations for the non-thermal emissivities across the Galaxy. Correspondingly, the line-of-sight integrated intensities for high-energy $\gamma$-rays and synchrotron emissions will encode characteristics of the discretised CR source activity and propagation. To illustrate, the top row of Fig. 2 shows the total $\gamma$-ray intensity summed over $\pi^{0}$-decay, bremsstrahlung, and IC emissions at $599.5 \mathrm{Myr}$ for the discretised solution at selected energies, and the bottom row shows the corresponding fractional residuals with the steady-state/smooth SA50 CR source density solution. The residuals show many features that 
require some care to interpret. About the Galactic plane toward the GC the line-of-sight integration path length is maximised and samples many under- and over-emissive regions, effectively averaging the fluctuations and generally obscuring the variations of the CR energy density distribution for the discretised solution. Hence the residuals are relatively flat in that direction. However, slightly out of the plane over the inner Galaxy the line-of-sight integration path length is lower and more details of discretised regions appear. But caution is still needed for the interpretation of the residuals. For example, the localised feature around $\sim 10^{\circ}-20^{\circ}$ below the $\mathrm{GC}$ is due to the combination of a nearby recently active region that is $\sim 1.5 \mathrm{kpc}$ distant $\sim 200-300 \mathrm{pc}$ out of the plane, as well as a complex $\sim 3 \mathrm{kpc}$ beyond through the same line of sight, with the relative contributions by different processes ( $\pi^{0}$-decay, bremsstrahlung, and IC) changing with energy. Around $\sim 1 \mathrm{GeV}$ it is the $\pi^{0}$-decay and bremsstrahlung from the nearby active region that is dominant but, with increasing energy, the IC emissions from the nearby region and beyond are more important. Over the inner Galaxy similar combinations of regions distributed along the line-of-sight produce the other residual features that are visible. Meanwhile toward the outer Galaxy about and just outside the plane there are residual features that have a slightly cleaner interpretation as being due to relatively nearby ( $\lesssim 1-3 \mathrm{kpc}$ ) complexes of discrete regions. The general progression of the $\pi^{0}$-decay and bremsstrahlung dominating residuals at lower energies while the IC takes over $\gtrsim 100 \mathrm{GeV}$ is also the case for these as well.
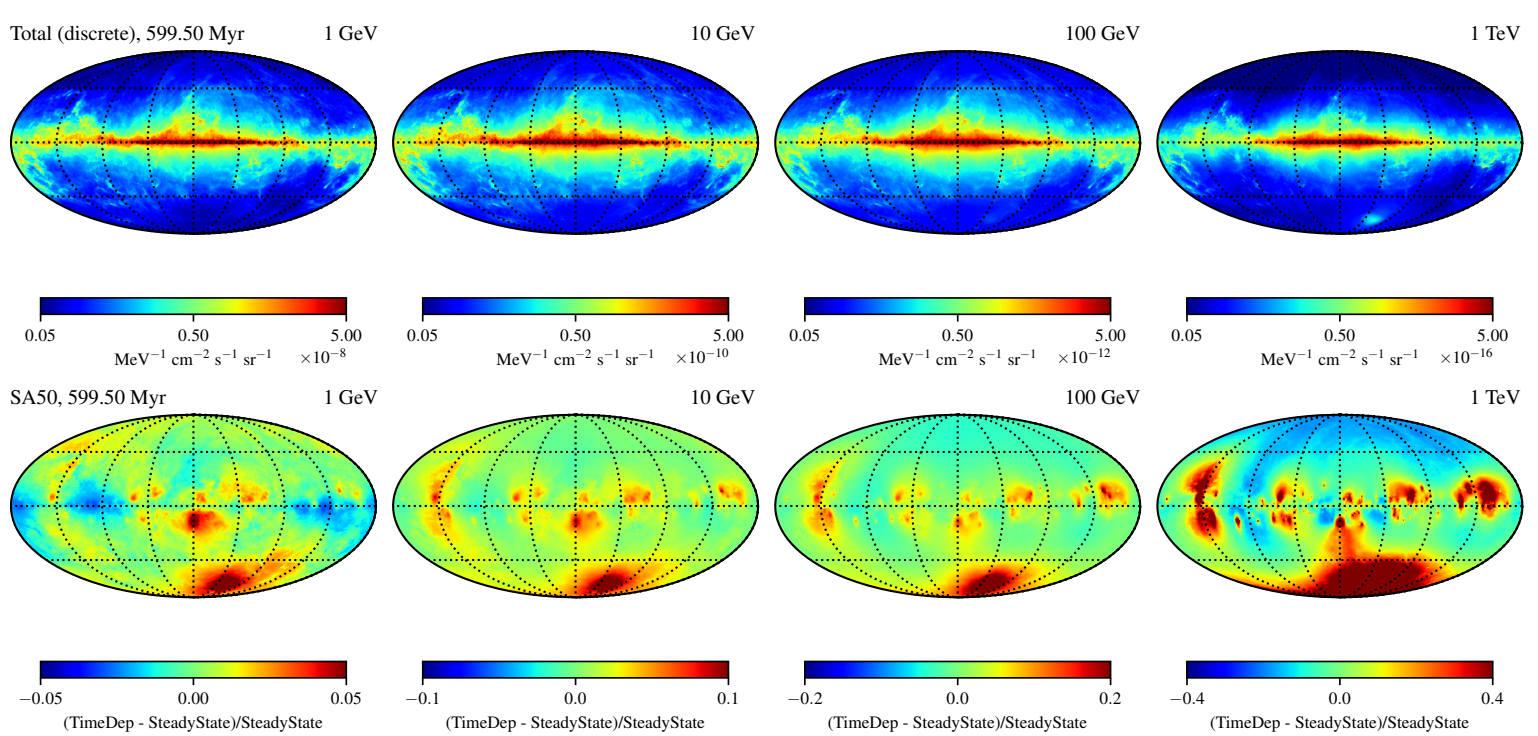

Figure 2: Total all-sky $\gamma$-ray intensity maps at selected energies at 599.5 Myr for the discrete source run (top row) and corresponding fractional residual maps at same energies using the SA50 steady-state/continuous solution.

Perhaps most intriguing is the appearance of an intense region at high southern latitudes in the total intensity map around $\sim 1 \mathrm{TeV}$, which produces a significant over intensity compared to that from the steady-state solution (Fig. 2 right-most, top/bottom row). The over intensity extends also to lower energies and is a very prominent feature that is broadly distributed. It is due to a nearby active source region that is a modest distance $\lesssim 1 \mathrm{kpc}$ outside of the plane. Correspondingly, the over intensity is mainly due to IC emissions, because the gas scale height out of the plane is much 
lower than that of the ISRF. The north/south asymmetry is a novel feature of the discretised model that is absent for the steady-state solution whose IC intensity predictions are symmetric about the plane. This is interesting because an explanation has proved elusive for the long-acknowledged asymmetry in the Fermi-LAT data at high Galactic latitudes, where the north polar region is more intense than the south by $\sim 20 \%$ around $1 \mathrm{GeV}$ with the discrepancy increasing with energy (see, e.g., Fig. 13 from [24]). It has the reverse sign to the difference between gas column density toward the respective polar regions. Based on the discretised modelling, the asymmetry may be the signature of an extended source of CRs at high northern latitudes. If the asymmetry has an IC origin there may also be implications for determination of the so-called 'isotropic' $\gamma$-ray background, which is comprised of extragalactic emissions that are too faint or diffuse to be resolved as well as residual Galactic foreground emissions that are approximately isotropic. Analyses of Fermi-LAT data $[25,26]$ have employed steady-state GALPROP-based foreground models that are symmetric about the Galactic plane to determine the isotropic background. The IC intensities at mid-to-high latitudes from these models are a major cause of systematic uncertainty for the Galactic foreground estimation. Correspondingly, modelling and analysis that does not account for possibly discretisation effects may induce biases in the derived isotropic background properties.
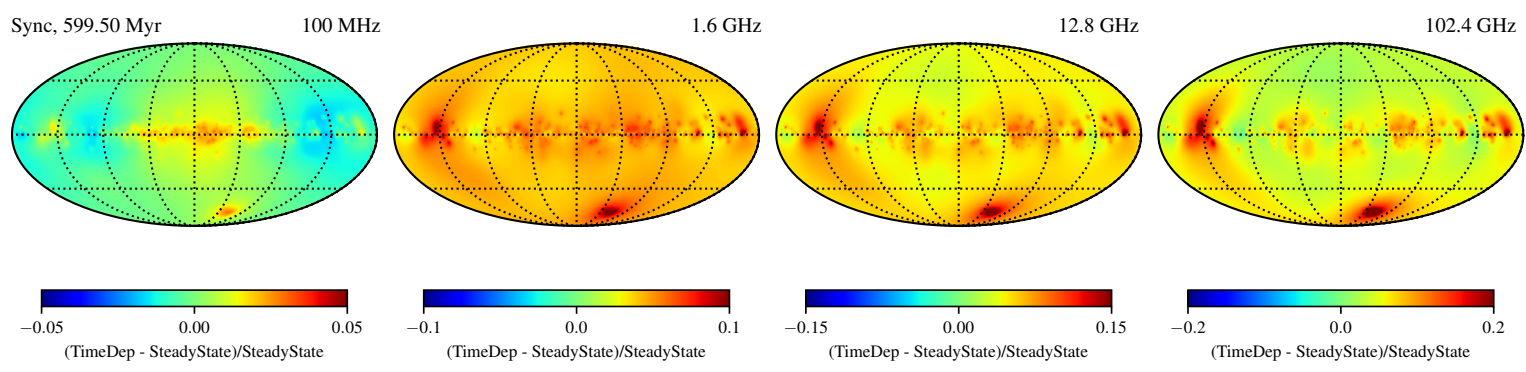

Figure 3: Fractional residual maps for synchrotron radiation at 599.5 Myr at selected frequencies.

The contributions by multiple processes complicates determination of the underlying CR source density distribution from high-energy $\gamma$-rays alone. Examining the synchrotron emissions may provide a useful way to resolve some ambiguities. Figure 3 shows the fractional residuals for the synchrotron intensities spanning $100 \mathrm{MHz}$ to $\sim 100 \mathrm{GHz}$ for the same time slice depicted in Fig. 2. The synchrotron residuals in fact display similar spatial characteristics to the IC fractional residuals (not shown) for energies $\sim 100 \mathrm{MeV}$ to $10 \mathrm{GeV}$, although there is not a precise mapping between the frequency and $\gamma$-ray energy ranges because of the different electromagnetic emission processes. Also, the difference between ISM target density distributions should be considered: the spiral patterns for the ISRF and magnetic field models are not the same and they have different energy distributions. But these mainly affect the predictions toward the inner Galaxy $\left(|l| \lesssim 45^{\circ}\right)$ and outside that region the synchrotron intensities can be a useful tool to enable disentangling the IC contribution to the high-energy $\gamma$-rays $\lesssim 10 \mathrm{GeV}$, particularly about the Galactic plane where the gas-related emissions are also strong.

To summarise, new features of the GALPROP code have been employed to efficiently calculate the evolution of a time/space-discretised CR source distribution from the equivalent continuous smooth density distribution. Comparison of the results for the CR intensities shows that their distribution across the Galaxy have fluctuations compared to the steady-state solution, even down to 
$\sim 1 \mathrm{GeV}$ energies. The corresponding non-thermal intensities for the discretised model also show many novel and non-trivial features compared to the equivalent steady-state solution. These may provide clues to the origin of puzzling features in high-energy $\gamma$-ray data, such as the north/south asymmetry, an explanation for which has proved elusive since its discovery.

\section{Acknowledgments}

GALPROP development is partially funded via NASA grant NNX17AB48G. Some of the results in this paper have been derived using the HEALPix [27] package.

\section{References}

[1] P. Predehl, et al. Proc. SPIE, p. 99051K, July, 2016.

[2] W. B. Atwood, et al., ApJ 697 (June, 2009) 1071-1102.

[3] H.E.S.S. Collaboration, $A \& A 612$ (Apr., 2018) A1.

[4] A. U. Abeysekara, et al., ApJ 843 (July, 2017) 40.

[5] T. A. Porter and R. J. Protheroe, Journal of Physics G Nuclear Physics 23 (Nov., 1997) 1765-1784.

[6] F. A. Aharonian and A. M. Atoyan, A\&A 362 (Oct., 2000) 937-952.

[7] R. E. Lingenfelter, Nature 224 (Dec., 1969) 1182-1186.

[8] R. Ramaty, D. V. Reames, and R. E. Lingenfelter, Physical Review Letters 24 (Apr., 1970) 913-916.

[9] J. C. Higdon and R. E. Lingenfelter, ApJ 582 (Jan., 2003) 330-341.

[10] R. Taillet, et al., ApJ 609 (July, 2004) 173-185.

[11] G. Bernard, et al., $A \& A 544$ (Aug., 2012) A92.

[12] P. Mertsch, JCAP 11 (Nov., 2018) 045.

[13] V. S. Ptuskin, et al., Adv. Sp. Res. 37 (2006) 1909-1912.

[14] A. W. Strong and I. V. Moskalenko, in vol. 587 of AIP Conf. Ser., pp. 533-537, Oct., 2001.

[15] A. W. Strong and I. V. Moskalenko, International Cosmic Ray Conference 5 (Aug., 2001) 1964-1967.

[16] T. A. Porter, G. Jóhannesson, and I. V. Moskalenko, ApJ 846 (Sept., 2017) 67.

[17] G. Jóhannesson, T. A. Porter, and I. V. Moskalenko, ApJ 856 (Mar., 2018) 45.

[18] A. Brandenburg and W. Dobler, Computer Physics Communications 147 (Aug., 2002) 471-475.

[19] M. S. Pshirkov, and et al., ApJ 738 (Sept., 2011) 192.

[20] G. Jóhannesson, T. A. Porter, and I. V. Moskalenko, ApJ 879 (jul, 2019) 91.

[21] V. S. Ptuskin, V. N. Zirakashvili, and A. A. Plesser, Adv. Sp. Res. 42 (Aug., 2008) 486-490.

[22] M. A. Malkov, et al., ApJ 768 (May, 2013) 73.

[23] L. Nava, et al., MNRAS 461 (Oct., 2016) 3552-3562.

[24] M. Ackermann, et al., ApJ 750 (May, 2012) 3.

[25] A. A. Abdo, et al., Physical Review Letters 104 (Mar., 2010) 101101.

[26] M. Ackermann, et al., ApJ 799 (Jan., 2015) 86.

[27] K. M. Górski, et al., ApJ 622 (Apr., 2005) 759-771. 